\title{
Contributions of academic institutions in high income countries to anesthesia and surgical care in low- and middle-income countries: are they providing what is really needed?
}

\author{
Shrividya Chellam, MD, DNB (D) - Lundeg Ganbold, MD, PhD • Anita Gadgil, MS, DNB $\cdot$ Sergelen Orgoi, MD, ScD • \\ Herman Lonnee, MbChB, FCA(SA) • Nobhojit Roy, MS, MPH, PhD • Adrian W. Gelb, MBChB, FRCPC
}

Received: 5 September 2018/Revised: 26 September 2018/Accepted: 27 September 2018/Published online: 20 November 2018

(C) Canadian Anesthesiologists' Society 2018

Data from the World Health Organization (WHO) suggest that by 2030, "the global burden of disease from malaria, tuberculosis, and HIV will be overshadowed by surgical diseases". ${ }^{1}$ Currently, $30 \%$ of the global disease burden is due to surgically correctable conditions with more than 30 million people facing catastrophic medical expenditure each year, defined as $>10 \%$ of total income or $40 \%$ of income after basic needs are met. ${ }^{2}$ Anesthesia mortality in high income countries (HIC) is very low, especially for healthy patients, at one in 100,000-200,000 cases, although major morbidity ranges between $3-16 \% .{ }^{1}$ Low and middle-

\section{S. Chellam, MD, DNB ( $\square)$.}

Department of Anesthesiology, BARC Hospital, Mumbai, India e-mail: shrivi74@gmail.com

L. Ganbold, $\mathrm{MD}, \mathrm{PhD}$

Department of Critical Care and Anesthesia, Mongolian,

National University of Medical Sciences, Ulaanbaatar, Mongolia

\section{A. Gadgil, MS, DNB}

Department of Surgery and WHO Collaborating Centre for Research on Surgical Care Delivery in LMICS, BARC Hospital, Mumbai, India

S. Orgoi, MD, ScD

Department of Surgery, Mongolian National University of

Medical Sciences, Ulaanbaatar, Mongolia

H. Lonnee, MbChB, FCA(SA)

Department of Anesthesia, St. Olavs' Hospital, Trondheim, Norway

N. Roy, MS, MPH, PhD

General Surgeon and Health Systems Consultant, Mumbai, India

\section{A. W. Gelb, MBChB, FRCPC}

Department of Anesthesia and Perioperative Care, University of California San Francisco, San Francisco, CA, USA income countries (LMIC) on the other hand, may have 1 death in 100-200 cases. Anesthesia can be challenging due to non-availability of equipment, such as pulse oximeters, anesthesia medications (including oxygen), and sometimes even basic amenities such as water and electricity. Vo et al. found in a study of 22 LMIC (including India) that $35 \%$ of healthcare facilities studied had no access to oxygen, $50 \%$ did not have continuous access to anesthesia machines or pulse oximeters, and $40-70 \%$ of medical devices and equipment were either broken, unused, or unfit for use. ${ }^{3}$ Lack of certified trained anesthesia providers compounds the problem. Indeed, HIC have 20-30 physician anesthesia providers per 100,000 individuals while LMIC have a ratio of one physician anesthesia provider per 100,000 individuals; in sub-Saharan Africa, the number drops to 0.1 per 100,000 population. ${ }^{4}$ There is a long tradition of interest by HIC academic anesthetic centres in aiding LMIC, and this interest continues to grow. ${ }^{5}$ A large proportion of this aid is in the form of shortterm medical missions, provision of equipment, and to a lesser extent, the training of personnel in LMIC. It is therefore appropriate to discuss whether the aid LMIC receive from HIC addresses the shortfall in anesthesia and surgery services adequately and appropriately. We present here the perspective of anesthesia and surgery providers in two Asian middle-income countries (India and Mongolia), both with large poor and underserviced populations.

\section{Charities and missions}

Short-term medical missions fulfill the immediate requirements for patient care, but are often cost ineffective and are unable to provide multidisciplinary long-term support to the very patients being operated upon. ${ }^{6}$ A hazard is that the mission doctors may not be 
sufficiently qualified or may be trainees who may cause serious medical complications in the absence of sufficient supervision. Team members may be taken by surprise by the extent of poverty, shortage of resources, and unintentionally insensitive to the local culture, language, or customs. ${ }^{7,8}$ Culture shock, and to a greater extent, reverse culture shock, are underestimated issues among healthcare workers. ${ }^{9}$ Reviews of data on elective medical missions have emphasized the need for sensitizing the team members to the cultural and contextual issues beforehand. They also note that the disease groups addressed by these missions are narrow, and mainly address cleft lip, palate, oral/dental health, and vaginal fistulae. ${ }^{8}$

Analysis of the issues with missions has identified common barriers to learning by local providers. These include resistance from either or both groups to change their modus operandi and practices. There is lack of support from superiors at the mission site, as well as longstanding habits that can be compounded by lack of materials. Facilitators to learning are evident (immediate) patient benefit in the eyes of the local providers, integrating local providers into the mission team, supportive leadership at the hospital, personal persistence, and the credibility and reputation of the training team. ${ }^{10}$ To learn from past mistakes (notably Haiti), and to prevent them in the future, the WHO developed a guideline document on "Classification and Minimum Standards for Foreign Medical Teams in Sudden Onset Disasters" in 2013 that emphasizes these and other important aspects of successful missions. ${ }^{11}$

Although short-term missions do not help in the long run, they still play a role in meeting the immediate and enormous surgical needs in LMIC. ${ }^{12}$ These missions would have a much greater impact with better pre-deployment planning and team preparation, detailed record keeping of the care delivered, documented patient follow-up and outcomes, publishing of analysis reports with cost per beneficiary, training conducted for local practitioners, and documentation of problems encountered, all of which could be shared with the local hospital. These processes can help avoid duplication or overlapping by similar groups. Transfer of skills by training the local workforce to sustain the mission's work would provide continued care and reduce further need to repeat the same mission thus allowing them to seek new opportunities elsewhere.

\section{Provision of equipment}

In 2000, the WHO published a comprehensive guide to appropriate medical donation. Donation of used "singleuse" or expired consumables raises substantial ethical issues that were also addressed by the WHO document. In simple colloquial terms, if it is not good enough for an operating room in a HIC then it should not be suitablefor an operating room anywhere. The World Federation of Societies of Anesthesiologists (WFSA) has recommendations on appropriate approaches to equipment purchase, but these apply equally well to equipment donation. ${ }^{13}$ Donors must consider whether there is dependable electricity; compressed gases supply; availability of spare parts; local expertise to install, service, or repair equipment (including manuals in the local language); and user training at the recipients' end to ensure benefit and utility. ${ }^{14}$ Lifebox Foundation (www. lifebox.org) has provided more than 15,000 pulse oximeters in 100 countries along with a multilingual manual, a video, an algorithm to manage hypoxia, power point presentations, and practice cases. The educational material is available free of charge in six languages covering different populations in various continents. ${ }^{15} \mathrm{~A}$ newer concept is "equipment brokerage" whereby equipment donation and suitably matched offers and requests are accepted online, together with volunteer expert suggestions. This may reduce equipment donations that are redundant and out of context for a particular LMIC. The WHO maintains a list of organizations that fulfill similar functions. ${ }^{16}$

\section{Training local personnel}

Lack of appropriately trained anesthesia providers creates a huge deficit in emergency surgical care, especially in obstetrics; this results in very high mortality and morbidity with approximately 300,000 women per year dying in labour. ${ }^{4}$ Although spinal anesthesia is usually considered safer than general anesthesia for obstetrics in LMIC, three-year data from South Africa suggest $79 \%$ of deaths directly related to anesthesia were due to spinal anesthesia resulting in hypotension or high spinal blockade. ${ }^{17}$ Training local teams to perform safe spinal anesthesia as well as other regional anesthesia techniques would help resolve this issue and promote safer anesthesia. Non-profit organizations and groups have designed and implemented working models for strengthening emergency surgical and obstetric care in LMIC (e.g., Association of Anaesthetists of Great Britain \& Ireland-WFSA Safe Obstetrics and Gynecology training). ${ }^{18}$ For long-term sustainability, educators have to be developed in LMIC by training the teachers in interactive teaching methods, allowing them to then teach basic and specialized anesthesia services to others. 


\section{Way forward}

The real need in LMIC is building local capacity by training and mentoring clinicians, together with technology and skill transfer by HIC academic centres, rather than short-term aid programs. The objectives of skill transfer should be based on careful assessment of local needs. Training should result in implementation of safe clinical practices coupled with quality control protocols. Training programs should include and integrate the whole surgical workforce-i.e., anesthesiologists, surgeons, operating room staff, and all other necessary team members, rather than focusing on a single group. The situational analysis tool, developed as part of Global Initiative for Essential and Emergency Surgical Care, has been included into the WHO Service Availability Mapping to help monitor surgical capacity as well as resource allocation and policy development. ${ }^{19}$ The WFSA has produced a detailed Anesthetic Facility Assessment Tool to document and monitor anesthetic capacity and includes the option to upload the data and have it analyzed for free (www.wfsahq.org/AFAT). The survey is based on the recently published WHO-WFSA International Standards for a Safe Practice of Anesthesia. ${ }^{20}$ The WHO Integrated Management of Essential and Emergency Surgical Care toolkit provides recommendations and guidance on policies, quality, safety, training, monitoring, and evaluation. This toolkit has been introduced in 35 countries as part of the global initiative for emergency and essential surgical care. Workforce retention can be ensured by an appropriate environment with robust infrastructure, adequate equipment, career growth, and financial remuneration. ${ }^{15}$ The role of academic centres in HIC is to facilitate and mentor the development of locally appropriate (national) surgical-obstetric-anesthesia planning that addresses these requirements. Final decisions about appropriate models and implementation must always rest with the in-country leadership.

Sending LMIC personnel to HIC for clinical or research training often results in them staying in HIC because of better career prospects. Training LMIC personnel locally leads to better local capacity building and a lower probability of relocation to HIC. An excellent example is WFSA's "Train the Trainer" courses that are run in the relevant country or region in conjunction with local partners. ${ }^{21}$ The intent is that these teachers will then in turn train others locally and thereby improve care and retain the workforce. Training programs should have an online component including refresher modules and online contact sessions with appropriately knowledgeable practitioners in HIC for regular discussion and follow-up. This will minimize the time trainees spend away from their place of work, while contact sessions give the necessary exposure to safe high-quality care in HIC. Programs could be organized as longer duration, well-spaced lectures, and modules for online training and shorter duration contact sessions. Available courses for training emergency medical team workers were summarized in a recent editorial. ${ }^{22}$

\section{Collaborative research}

In addition to training and capacity building, collaborative research can lead to locally able innovations, and sustainable as well as acceptable solutions. Universities in HIC have been partnering with academic centres in LMIC for exchange of skills, expertise, and creation of collaborative research programs. ${ }^{23}$ Research conducted with international collaboration often ends when overseas researchers return home. Hence, sustained online collaborations and the creation of centres of excellence in LMIC build local research capacity. Many HIC countries have award programs for collaborative research projects, which contribute by giving access to the better HIC research methodology but applied in the LMIC context.

Mongolia serves as an excellent example of how HIC can help effect huge sustainable changes. The Lancet Commission on Global Surgery states that to meet the world's surgical targets, $80 \%$ coverage of essential surgical and anesthesia services is required along with the provision of 5,000 procedures per 100,000 population by 2030 . The WFSA, Bangkok Anesthesia Regional Training Centre, and anesthesiologists' societies from Switzerland and Australia, have worked with the WHO collaboration centres in Mongolia for over a decade. They have contributed to the sustained improvement in surgery and anesthesia care by training the local doctors and nurses, establishing residency programs, collaborative research and publications, and setting up standard of care hospitals and intensive care units. This collaboration has already achieved and overshot the Lancet Commission 2030 target in terms of access to surgery, surgical workforce density per 100,000 , surgical volume per 100,000 , and surgical complications rates. ${ }^{24}$ Thus, enhancing the local service capacity seems a viable long-term solution.

In conclusion, HIC efforts need to be directed towards better planned and organized mission trips with integration among mission groups to prevent overlapping visits and greater cultural sensitivity. Such missions need to remember that they only meet the short-term, narrowly focused needs of LMIC. We ask such non-governmental agencies to consider including into their often-repeated (educational) visits, activities for anesthesia, surgery, and nursing that help build infrastructure. In addition, and of greater importance, are the long-term needs that require academic centres in HIC to commit to sustainable training 
programs focused on local capacity building, guidance and assistance in acquisition and use of equipment, and workforce retention. Addressing these longer term needs should be integrated into the activities of short-term missions.

\section{Contributions des établissements d'enseignement des pays à haut revenu aux soins anesthésiques et chirurgicaux dans les pays à revenus intermédiaires et faibles : fournissent-ils ce qui est vraiment nécessaire?}

Les données de l'Organisation mondiale de la santé (OMS) suggèrent que d'ici 2030, «la charge mondiale de morbidité due au paludisme, à la tuberculose, et au VIH sera éclipsée par les maladies chirurgicales ». ${ }^{1}$ Actuellement, $30 \%$ de la charge mondiale de morbidité est due à des affections qui peuvent être résolues par la chirurgie; plus de 30 millions de personnes sont confrontées chaque année à des dépenses médicales catastrophiques, c'est-à-dire plus de $10 \%$ de leur revenu total ou $40 \%$ du revenu après déduction des dépenses pour les besoins fondamentaux. ${ }^{2}$ La mortalité anesthésique dans les pays à revenu élevé (PRÉ) est très faible, en particulier pour les patients en bonne santé, de l'ordre d'un décès pour cent à deux cent mille cas, bien que la morbidité majeure soit de $3 \%$ à $16 \%$. ${ }^{1}$ Les pays à revenu intermédiaire ou faible (PRIF) souffrent d'un manque de disponibilité en équipement, tels que les oxymètres, les médicaments anesthésiques (y compris l'oxygène) et parfois même de l'absence de commodités de base comme l'eau et l'électricité. Dans une étude portant sur 22 PRIF (y compris l'Inde), Vo et coll. ont trouvé que $35 \%$ des établissements étudiés n'avaient pas d'accès à l'oxygène, $50 \%$ n'avaient pas d'accès continu aux machines d'anesthésie ou aux oxymètres de pouls et $40 \%$ à $70 \%$ des dispositifs ou équipements médicaux étaient brisés, inutilisés ou inadaptés aux besoins. ${ }^{3}$ Le manque de prestataires formés en anesthésie complique le problème. En fait, les PRÉ disposent de 20 à 30 médecins prestataires d'anesthésie pour 100000 habitants tandis que dans les PRIF, le ratio est d'un pour 100000 ; en Afrique subsaharienne, le nombre tombe à 0,1 pour 100000 habitants. $^{4} \quad$ Les centres anesthésiques universitaires des PRÉ ont une longue tradition d'aide des PRIF et cet intérêt continue de croitre. ${ }^{5}$ Une grande partie de cette aide se présente sous forme de missions médicales de courte durée, de fourniture d'équipement et dans une moindre mesure - de formation des personnels dans les PRIF. Il est donc tout à fait indiqué de se demander si l'aide que les PRÉ apportent aux PRIF répond au manque de services d'anesthésie et de chirurgie de façon adéquate et appropriée. Nous présentons ici le point de vue des prestataires de services en anesthésie et chirurgie dans deux pays asiatiques à revenu intermédiaire (l'Inde et la Mongolie) qui ont tous les deux de grandes populations pauvres et démunies sur le plan des services.

\section{Organisations caritatives et missions}

Les missions médicales à court terme répondent aux besoins immédiats des soins aux patients, mais elles sont souvent non rentables et sont incapables de fournir un soutien multidisciplinaire à long terme à ces mêmes patients qui ont été opérés. ${ }^{6}$ L'un des risques et que les médecins de la mission ne soient pas suffisamment qualifiés ou soient même des stagiaires, ce qui peut être la cause de complications médicales graves en l'absence de supervision adéquate. Les membres de l'équipe peuvent être pris par surprise devant l'étendue de la pauvreté, du manque de ressources et être involontairement insensibles à la culture, à la langue ou aux coutumes locales. ${ }^{7,8} \mathrm{Le}$ choc des cultures, et dans une plus grande mesure le choc des cultures inversé sont des problèmes sous-estimés parmi les travailleurs du domaine de la santé. ${ }^{9}$ Les analyses des données de missions médicales non urgentes ont insisté sur le besoin de sensibiliser préalablement les membres de l'équipe aux problèmes culturels et contextuels. Elles notent également que les groupes de maladies abordés par ces missions sont restreints et concentrés principalement sur les fentes labiales, les fentes palatines, la santé buccale/dentaire et les fistules vaginales. ${ }^{8}$

L'analyse des problèmes posés par les missions a identifié des obstacles communs à l'apprentissage par les prestataires locaux. Il s'agit notamment de la résistance de l'un ou des deux groupes à modifier son mode opératoire et ses pratiques. Il y a aussi un manque de soutien de la part des supérieurs sur le site de la mission ainsi que des habitudes de longue date qui peut être amplifiée par le manque de matériel. Les facilitateurs de l'apprentissage apportent des avantages évidents (immédiats) pour le patient aux yeux des prestataires locaux, en intégrant les prestataires locaux dans l'équipe de la mission, avec un leadership encourageant à l'hôpital, la persistance du personnel, ainsi que la crédibilité et la réputation de l'équipe assurant la formation. ${ }^{10}$ Pour apprendre des 
erreurs du passé (en particulier à Haïti) et éviter qu'elles ne se reproduisent à l'avenir, l'OMS a élaboré des lignes directrices sur la «Classification et normes minimales de prestation pour les équipes médicales étrangères confrontées à une catastrophe subite » (Classification and Minimum Standards for Foreign Medical Teams in Sudden Onset Disasters) en 2013 qui insistent sur ces points ainsi que sur d'autres aspects importants pour la réussite des missions. ${ }^{11}$

Même si les missions à court terme ne sont pas utiles à long terme, elle joue tout de même un rôle pour répondre aux besoins chirurgicaux énormes et immédiats des PRIF. ${ }^{12}$ Ces missions auraient un beaucoup plus grand impact avec une meilleure planification et préparation de l'équipe avant le déploiement, des dossiers détaillés des soins fournis, du suivi et de l'évolution des patients, la publication de rapports d'analyse avec leur coût par bénéficiaire, la formation apportée aux praticiens locaux, et une documentation des problèmes rencontrés; tout cela pouvant être partagé avec l'hôpital local. Ces processus peuvent contribuer à éviter une duplication ou un chevauchement entre des groupes similaires. Le transfert des compétences par la formation du personnel local visant à soutenir le travail de la mission pourrait fournir des soins continus et réduire la nécessité d'avoir à répéter la même mission, permettant de rechercher de nouvelles opportunités ailleurs.

\section{Fourniture d'équipements}

L'OMS a publié en 2000 un guide complet sur les dons médicaux appropriés. Le don de produits consommables à « usage unique » ayant servi ou dont la date de péremption est dépassée pose des problèmes éthiques substantiels qui ont été également abordés par le document de l'OMS. Dit en termes simples, si ce n'est pas assez bon pour une salle d'opération dans un PRÉ, alors cela ne devrait pas pouvoir servir dans une salle d'opération ailleurs dans le monde. La Fédération mondiale des sociétés d'anesthésiologistes (WFSA) propose des recommandations sur les approches convenables concernant l'achat d'équipements, mais qui s'appliquent également bien aux dons d'équipements. ${ }^{13}$ Les donateurs doivent prendre en compte l'existence d'une alimentation électrique fiable, la disponibilité de gaz comprimé, de la disponibilité de pièces de rechange, de l'expertise locale pour l'installation, l'entretien ou la réparation de l'équipement (y compris l'existence de manuels dans la langue locale), et la formation de l'utilisateur du côté des destinataires pour garantir un bénéfice et une utilité. ${ }^{14} \mathrm{La}$ fondation Lifebox Fondation (www.lifebox.org) a fourni plus de 15000 oxymètres de pouls dans 100 pays avec un manuel en plusieurs langues, un vidéo, un algorithme de gestion de l'hypoxie, des présentations en format PowerPoint et des exemples pratiques. Le matériel éducatif est disponible gratuitement dans six langues, couvrant différentes populations sur plusieurs continents. ${ }^{15}$ Un concept plus récent est «le courtage d'équipement » par lequel le don d'équipement et des offres et demandes correctement appariées sont acceptées en ligne, en même temps que les suggestions d'experts bénévoles. Cela pourrait limiter les dons d'équipements redondants et inappropriés dans le contexte d'un PRIF particulier. L'OMS tient une liste des organismes qui assurent des fonctions similaires. ${ }^{16}$

\section{Formation du personnel local}

L'absence de prestataires en anesthésie correctement formés crée un énorme déficit pour les soins chirurgicaux d'urgence, notamment en obstétrique; cela entraine une mortalité et une morbidité très élevées avec environ 300000 femmes mourant chaque année au cours de l'accouchement. ${ }^{4}$ Même si la rachianesthésie est habituellement jugée plus sûre que l'anesthésie générale pour l'obstétrique dans les PRIF, des données sur trois ans provenant d'Afrique du Sud suggère que $79 \%$ des décès directement liés à l'anesthésie étaient dus à une rachianesthésie ayant provoqué une hypotension ou un bloc haut. ${ }^{17} \mathrm{La}$ formation des équipes locales à la réalisation d'une rachianesthésie sécuritaire ainsi qu'aux autres techniques d'anesthésie régionale contribuerait à résoudre ce problème et à promouvoir une anesthésie plus sûre. Des organismes et des groupes sans but lucratif ont conçu et mis en œuvre des modèles de travail pour le renforcement des soins chirurgicaux et obstétricaux d'urgence dans les PRIF (p. ex. l'Association des anesthésiologistes de Grande-Bretagne et d'Irlande Formation sécuritaire en gynécologie et obstétrique de la FMSA). ${ }^{18}$ Pour une pérennité à long terme, des éducateurs doivent être mis en place dans les PRIF en formant les professeurs aux méthodes d'enseignement interactif leur permettant alors d'enseigner à d'autres les techniques de base et spécialisées en anesthésie.

\section{Pour aller de l'avant}

Le vrai besoin des PRIF est de bâtir des capacités locales par la formation et le mentorat des cliniciens, ainsi que par le transfert de technologies et de compétences par les centres universitaires des PRÉ, plutôt que par des programmes d'aide à court terme. Les objectifs de transferts des compétences doivent reposer sur une évaluation méticuleuse des besoins locaux. La formation 
doit aboutir à la mise en œuvre de pratiques cliniques sécuritaires couplées à des protocoles de contrôle de la qualité. Les programmes de formation doivent inclure et intégrer la totalité des effectifs chirurgicaux, c'est-à-dire les anesthésiologistes, les chirurgiens, le personnel de salle d'opération et tous les autres collaborateurs nécessaires, plutôt que de se concentrer sur un seul groupe. L'outil d'analyse contextuelle développé dans le cadre de l'Initiative mondiale pour les soins essentiels et chirurgicaux urgents (GIEESC) a été inclus par l'OMS dans la cartographie des services disponibles (SAM) pour contribuer à la gestion des capacités chirurgicales ainsi qu'à la répartition des ressources et à l'élaboration de politiques. ${ }^{19}$ La FMSA a produit un Outil d'évaluation des établissements d'anesthésie détaillé pour documenter et suivre la capacité anesthésique; il inclut une option permettant de télécharger des données et de les faire analyser gratuitement (www.wfsahq.org/AFAT). L'enquête repose sur les Normes internationales pour une pratique sécuritaire de l'anesthésie récemment publiées par l'OMS et la FMSA. ${ }^{20}$ La trousse à outils de l'OMS «Integrated Management of Essential and Emergency Surgical Care » donne des recommandations et des conseils sur les politiques à adopter, la qualité, la sécurité, le suivi et l'évaluation. Cette trousse à outils a été présentée dans 35 pays dans le cadre de l'initiative globale pour les soins d'urgence et chirurgicaux essentiels. La fidélisation des effectifs peut être assurée grâce à un environnement approprié dans une infrastructure robuste, avec un équipement adapté, une progression de carrière et une rémunération financière. ${ }^{15}$ Le rôle des centres universitaires des PRÉ est de faciliter et d'encadrer le développement d'une planification chirurgicale-obstétricale-anesthésiste (nationale) correspondant aux besoins locaux et répondant à ces exigences. Les décisions finales sur les modèles appropriés et leur mise en œuvre doivent toujours reposer sur le leadership du pays concerné.

Le personnel des PRIF envoyé vers les pays à haut revenu pour une formation clinique ou en recherche reste souvent dans ces derniers en raison de meilleures perspectives de carrières. Former localement les personnels des PRIF débouche sur une meilleure capacité de développement local et à une moindre probabilité de relocalisation dans les PRÉ. On trouve un excellent exemple dans les cours de la FMSA «Train the Trainer » (Former le formateur) qui ont lieu dans le pays ou la région concernée en coopération avec les partenaires locaux. ${ }^{21}$ Le but est que ces enseignants deviennent à leur tour les formateurs locaux d'autres professionnels et améliorent ainsi les soins, fidélisant aussi les effectifs. Les programmes de formation doivent inclure une composante en ligne avec des modules de mise à jour des connaissances et des sessions de communications avec des praticiens compétents dans les PRÉ pour des discussions régulières et un suivi. Cela réduira au minimum le temps d'absence des stagiaires hors de leur lieu de travail tandis que les sessions de communications procureront une exposition nécessaire aux soins sécuritaires de haute qualité des PRÉ. Les programmes pourraient être organisés sur de plus longues durées, avec des cours bien espacés, des modules pour la formation en ligne et des sessions de communications plus brèves. Les cours disponibles pour la formation des équipes médicales d'urgence ont été résumés dans un récent éditorial. ${ }^{22}$

\section{Recherche collaborative}

En plus de la formation et de la capacité de développement, une recherche collaborative peut déboucher sur des innovations localement utilisables ainsi que sur des solutions durables et acceptables. Les universités des PRÉ ont travaillé en partenariat avec les centres universitaires des PRIF pour échanger des compétences, de l'expertise et créer des programmes de recherche collaborative. ${ }^{23} \mathrm{La}$ recherche menée avec une collaboration internationale prend souvent fin quand les chercheurs d'outremer retournent chez eux. Par conséquent, la persistance de collaborations en ligne et la création de centres d'excellence dans les PRIF bâtissent une capacité locale de recherche. De nombreux pays à haut revenu ont des programmes de subventions pour les projets de recherche collaborative qui contribuent à donner accès à la meilleure méthodologie de recherche des PRÉ appliquée au contexte des PRIF.

La Mongolie est un excellent exemple de la façon dont les PRÉ peuvent contribuer à réaliser des changements durables majeurs. La «Lancet Commission on Global Surgery » indique que pour atteindre les cibles chirurgicales mondiales, une couverture de $80 \%$ des services chirurgicaux et anesthésiques essentiels est nécessaire ainsi que la réalisation de 5000 procédures par 100000 habitants d'ici 2030. Le Centre de formation régional en anesthésie de Bangkok de la FMSA et les sociétés d'anesthésiologistes de Suisse et d'Autriche ont travaillé avec les centres de collaboration de l'OMS en Mongolie depuis plus d'une décennie. Ils ont contribué à l'amélioration continue des soins chirurgicaux et anesthésiques en formant des médecins et infirmiers locaux, en créant des programmes de résidence, une recherche collaborative et des publications, et en mettant sur pied des hôpitaux et unités de soins critiques répondant aux normes de soins. Cette collaboration a déjà atteint et même dépassé la cible 2030 de la Lancet Commission en termes d'accès à la chirurgie, de densité des effectifs chirurgicaux et des volumes d'interventions par 
100000 habitants, et des taux de complications chirurgicales. $^{24}$ Ainsi, favoriser les services locaux semble une solution viable à long terme.

Pour conclure, les efforts des pays à haut revenu doivent être dirigés directement vers des voyages de missions mieux planifiées et organisées avec une intégration entre les groupes de mission pour éviter le chevauchement de visites et une plus grande sensibilité aux différences culturelles. Ces missions ne doivent pas oublier qu'elles ne répondent qu'à des besoins à court terme étroitement sélectionnés des PRIF. Nous demandons que ces organismes non gouvernementaux envisagent d'inclure dans leurs visites (éducatives) souvent répétées des activités pour l'anesthésie, la chirurgie et les soins infirmiers qui contribuent au développement des infrastructures. De plus, et plus important encore, les centres universitaires des PRÉ doivent s'engager à long terme envers des programmes de formation durables centrés sur le développement des capacités locales, l'orientation et l'aide pour l'acquisition et l'utilisation d'équipement, enfin pour la fidélisation des effectifs. Répondre à ces besoins à long terme doit faire partie des activités des missions à court terme.

Conflicts of interest Adrian W. Gelb is Secretary of the World Federation of Societies of Anesthesiologists.

Editorial responsibility This submission was handled by Dr. Hilary P. Grocott, Editor-in-Chief, Canadian Journal of Anesthesia.

Funding None declared.

Conflits d'intérêts Adrian W Gelb est Secrétaire de la Fédération mondiale des sociétés d'anesthésiologistes (FMSA/WFSA).

Responsabilité éditoriale Cet article a été traité par le $\mathrm{D}^{\mathrm{r}}$ Hilary $\mathrm{P}$. Grocott, Rédacteur en chef, Journal canadien d'anesthésie.

Financement Aucun déclaré.

\section{References}

1. Boggs $S D$, Chee NW. Anesthesia disparities between high-income countries and low-and middle-income countries: providers, training, equipment, and techniques. In: Roth R, Frost EA, Gevirtz C, Atcheson C (eds). The Role of Anesthesiology in Global Health - A Comprehensive Guide. Springer; 2015: 41-59. Available from URL: https://www.uclahealth.org/anes/workfiles/ about-us/The-Role-of-Anesthesiology-in-Global-Health.pdf (accessed October 2018).

2. Meara JG, Leather AJ, Hagander L, et al. Global surgery 2030: evidence and solutions for achieving health, welfare, and economic development. Lancet 2015; 386: 569-624.

3. Vo D, Cherian MN, Bianchi S, et al. Anesthesia capacity in 22 low and middle income countries. J Anesth Clin Res 2012; DOI: https://doi.org/10.4172/2155-6148.1000207.
4. World Federation of Societies of Anaesthesiologists. World Anaesthesiology Workforce. Available from URL: https://www. wfsahq.org/workforce-map (accessed October 2018).

5. Evans FM, Mallepally NR, Dubowitz G, Vasilopoulos T, McClain $C D$, Enneking $K$. Factors influencing anesthesia residency selection: impact of global health opportunities. Can J Anesth 2016; 63: 674-81.

6. Evans FM, Nabukenya MT. Con: Pure service delivery is no longer needed in global surgical missions. Can J Anesth 2017; 64: 353-7.

7. DeCamp M, Lehmann LS, Jaeel P, Horwitch C, Ethics Professionalism and Human Rights Committee. Ethical obligations regarding short-term global health clinical experiences: an American College of Physicians position paper. Ann Intern Med 2018; 168: 651-7.

8. Martiniuk AL, Manouchehrian M, Negin JA, Zwi AB. Brain gains: a literature review of medical missions to low and middle-income countries. BMC Health Serv Res 2012; 12: 134.

9. Frazier MK, Kasten S. Reverse culture shock: the Purdue Kenya partnership. Purdue J Serv Int Engagem 2015; DOI: https://doi. org/10.5703/1288284315688.

10. White $M$, Close $K$. Lasting impact: insights from a surgical mission-based mentoring training programme in the Republic of Congo. BMJ Global Health 2016; 1: e000102.

11. Norton I, von Schreeb J, Aitken P, Herard P, Lajolo C. Classification and minimum standards for foreign medical teams in sudden onset disasters. World Health Organization 2013. Available from URL: http://www.who.int/hac/global_ health_cluster/fmt_guidelines_september2013.pdf (accessed October 2018).

12. White $M C$. Pro: Pure service delivery is still needed in global surgery missions. Can J Anesth. 2017; 64: 347-52.

13. World Federation of Societies of Anaesthesiologists Safety and Quality. Available from URL: www.wfsahq.org/our-work/safetyquality (accessed October 2018).

14. Gatrad AR, Gatrad S, Gatrad A. Equipment donation to developing countries. Anaesthesia 2007; 62(Suppl 1): 90-5.

15. Enright A, Merry A, Walker I, Wilson I. Lifebox: a global patient safety initiative. A A Case Rep 2016; 6: 366-9.

16. World Health Organization. Organisations working on donations of medical equipment. Available rom URL: http://www.who.int/ medical_devices/management_use/donation_org_roles.pdf (accessed October 2018).

17. Reed A, Mumba JM, Dyer R. A spotlight on obstetric anesthesia in the developing world: finally getting the attention it deserves. Anesth Analg 2015; 120: 1179-81.

18. World Federation of Societies of Anaesthesiologists. Safer Anaesthesia From Education (SAFE). Available from URL: https://www.wfsahq.org/wfsa-safer-anaesthesia-from-educationsafe (accessed October 2018).

19. Bickler SW, Spiegel D. Improving surgical care in low- and middle-income countries: a pivotal role for the World Health Organization. World J Surg 2010; 34: 386-90.

20. Gelb AW, Morriss WW, Johnson W, Merry AF, International Standards for a Safe Practice of Anesthesia Workgroup. World Health Organization-World Federation of Societies of Anesthesiologists (WHO-WFSA) International Standards for a Safe Practice of Anesthesia Can. J Anesth 2018; 65: 698-708.

21. World Federation of Societies of Anaesthesiologists. Train the Trainer. Available from URL: http://monitor.pubs.asahq.org/ article. aspx ?articleid=2571369 (accessed October 2018).

22. Hemmings HC Jr, Hunter JM. Landmarks in UK anaesthesia. Br J Anaesth 2017; 119: 1073-4.

23. Macfarlane SB, Agabian N, Novotny TE, Rutherford GW, Stewart CC, Debas HT. Think globally, act locally, and collaborate 
internationally : global health sciences at the University of California, San Francisco. Acad Med 2008; 83: 173-9.

24. Sergelen O, Ganbold L, Bat-Orgil.B. Mongolian National University of Medical Sciences. WHO Collaborating Centre for
Emergency and Essential Surgical Care. WHO EESC Collaborating Center in Mongolia/MOG-1. Available from URL: http://www.wpro.who.int/whocc_forum/agenda/mog1_ emergency_essential_surgical_care.pdf (accessed October 2018). 